\title{
Crowdsourced Recommender System
}

\section{Montebello, Matthew and Mallia-Milanes, Mario}

Faculty of Information and Communication Technology, Department of Artificial Intelligence, University of Malta, Malta.

\begin{abstract}
The use of artificially intelligent techniques to overcome specific shortcomings within e-learning systems is a well-researched area that keeps on evolving in an attempt to optimise such resourceful practices. The lack of personalization and the sentiment of isolation coupled with a feeling of being treated like all others, tends to discourage and push learners away from courses that are very well prepared academically and excellently projected intellectually. The use of recommender systems to deliver relevant information in a timely manner that is specifically differentiated to a unique learner is once more being investigated to alievate the e-learning issue of being impersonal. The application of such a technique also assists the learner by reducing information overload and providing learning material that can be shared, criticized and reviewed at one's own pace. In this paper we propose the use of a fully automated recommender system based on recent AI developments together with Web 2.0 applications and socially networked technologies. We argue that such technologies have provided the extra capabilities that were required to deliver a realistic and practical interfacing medium to assist online learners and take recommender systems to the next level.
\end{abstract}

Keywords: Artificial Intelligence; e-Learning; Recommender System; Collaborative; Crowdsource. 


\section{Introduction}

E-Learning may seem to be a new idea, but in actual fact it was preceded by what used to be distance learning or correspondence learning. Its roots can be traced back to the $18^{\text {th }}$ century whereby material was distributed to students, not in close proximity to the teaching source, via post (Montebello M. , 2018). This idea evolved over the years and the use of the Internet facilitated distribution. With the advent of Web 2.0 the capability to display multimedia became available and thus learning material became feature rich. It can be said, at this point, that students were not actually bound by proximity or time anymore. Which at face value is a very significant factor as it reduces cost and can open up courses to larger audiences. In theory anyone all over the world can attend.

Sadly e-learning and its various mutations did not really achieve the success that was thought would be reaped. In many instances the dropout rate from courses was extremely high (Rivard, 2013). The reason for this was not immediately apparent. It soon became evident that the pedagogy never changed since the inception of the remote learning concept. Design materials, package them in a convenient way and distribute them to as many people as possible. This one-size-fits-all scenario really lived on through the ages, and survived different dissemination media. So, when we ask the question what was the impact of Information Technology on learning the answer is difficult to ascertain.

Currently many institutes like Coursera and Udemy deliver a variety of on-line courses. Material is in no way customized to suit individual needs, learning styles or abilities. A mass-produced package is delivered. This is very convenient for the institution, very cost effective, but not learner centric.

So, having described the scenario so far, it would be appropriate to define an important point at this stage; the meaning of learning. Chen and Wei state this properly as "learning is an active, interactive and social process" (Chen \& Wei, 2004). A look at this definition it becomes clear that distance learning is not conducive to it. This because learning requires synergy between a number of activities. Each activity facilitates the uptake of new skills. Ultimately learning can also be seen as the activity carried out by a person to acquire new skills or knowledge, even by interacting with others. So, there should be some sort of conduit between teacher, learner and peers. This interaction places the learner at the centre of the knowledge transfer activity.

Questions to solve this problem start to become apparent, namely:

- What makes a student want to study?

- What engages a student?

- Can a software system be designed to solve this issue? 


\section{The E-learning Concept}

As a concept e-learning means different things to different people. In this paper it is taken to mean the use of technology to facilitate learning. In other words, the act of transcribing manual notes into a digital format is not accepted as e-learning. Commonly e-Learning is delivered over the Internet and provides interactivity with the student, having materials focused on the student (Nicholson, 2007).

Early in the $20^{\text {th }}$ Century John Dewey, and later on Carl Rogers, insisted that education should focus on the experience of the learner. Many criticize modern e-learning tools with their incapacity to do this. Despite the benefit of having material shared globally at one go the issue of having personalized material still remains. No two learners are alike, and hence the task of assimilating material to each individual still needs to be handled properly. The environment students are placed in when taking an on-line course is that of autonomy and self-direction. And the user, the student, is not at the centre of the equation (Garrison, 2011).

\subsection{Tools for e-Learning}

There are a plethora of tools that are available for the content designer today. E-mail, presentation packages, video material, content management systems, social media and blogs practically cover the whole spectrum. But in taking a closer look at these tools it can be noticed that they cannot scale well to a user's needs. Collaboration is limited and may not be in real-time either. Thus, one of the most important elements, that of peer collaboration, would be conspicuous by its absence (Mallia-Milanes \& Montebello, 2017).

Collaboration uniquely helps the development of the identity of the learner by allowing him to interact with an environment that projects roles and values on that person. Conversely by limiting the ability to share and interact with others would induce demotivation and make a student leave a course. This is reflected in the low completion rates experienced on elearning courses (Rivard, 2013).

\subsection{Taking the Next Step}

So effectively what is needed is a new approach to e-learning rather than the technology. The approach has to be flexible and scalable. This to adjust to communities of users who would wish to take up learning a new skill within their own time, and at their own pace. So, we need a system that can adapt to a student and as yet leave room for flexibility to allow the student to experience and absorb his way through the subject.

Artificial Intelligence techniques can be used to fill in this role (Montebello M. , 2014). Rather than delivering a whole software package a team of agents, each specialized in its own realm, can be deployed with the aim of assisting the student. These groups of agents 
would collaborate to deliver a seamless output to the student that makes sense to him. Moreover, output needs to be delivered in a timely fashion. Montebello (2018) puts forward three models which can assist, namely:

- Capture of user's interests;

- $\quad$ Profile of the user;

- Recommender systems.

All three are necessary and would be able to feature as a group of agents within a system capable of delivering the desired output.

The first and second point of the list could be managed in a number of ways. Either by asking a potential student questions to get an idea of what he needs and what are his abilities. On the other hand, the agent system can surreptitiously form a profile and a list of interests by "observing” the user's behaviour. Naturally a plethora of ethical issues would emerge of each approach. This would demand a study in its own. AI systems are insensitive to people's behaviour, so unfettered reporting and profiling can be uncomfortable, inappropriate, or even illegal.

\subsection{Recommender Systems}

Recommender systems have come into play for a number of applications. Their main stay has been in sales websites where a client is offered items that he may also like. Netflix, Amazon and also YouTube are typical examples of such websites. So, in essence the aim of a recommender system is twofold; to induce sales, and to reduce information overload (Jannach \& Zanker, 2011). The techniques are rooted in information retrieval and filtering.

There are two basic approaches to recommender systems, namely, Collaborative filtering (Jannach \& Zanker, 2011), and Similarity indexing (Jannach \& Zanker, 2011). In collaborative filtering, the algorithm has to match a closest neighbour. This is basically done by comparing the buying patterns of the current user, with that to others who have purchased similar items in the past. Recommendations are then put forward on the basis of the likelihood that the current user would probably have the same taste as other users with similar patterns. The similarity indexing approach rates and marks each product on offer. When a user chooses a particular product then others similar to it are put forward as a recommendation. Both approaches have issues. Typically, the collaborative filtering approach suffers from cold start. How shall we compare if we have very little or no data to go on? On the other hand, a similarity indexing solution is computationally intensive. Imagine a situation with thousands of users on-line at the same time each going through many items available for sale. 


\section{Adaptation to e-Learning}

As discussed earlier on, one of the main issues of current e-learning systems are that they suffer from the lack of personalization. One way to jump over this hurdle is to "cocoon" the student within an automated learning environment that recommends and coaches learners with adequate resources and personlaised suggestions. This would be made up of a teacher, peers, and material to draw on. Material can be crowdsourced, by having many input points feeding the student with his necessities (Montebello M. , 2015).

The point of the recommender system in this setup would be to prevent a cognitive overload by supplying too much in too little time to the user. Moreover, the system would have to deal with information relevance apart from its timeliness (Jannach \& Zanker, 2011).

\subsection{Hybrid Approach}

So, we are proposing a system (Figure 1) whereby data is brought up to the student which is relevant to his learning needs. This can be done in a straightforward way as if one is buying items of the Internet. In addition to the material, the recommender system will have to match the current user with others who have similar needs or experiences, and also a teacher who has a declared expertise in the subject matter. Crowdsourced material, experts and peers can be pulled together on the fly to create an environment similar to a class, but without the boundaries imposed by space and time. This would give the student the opportunity to share, experience and scaffold though material till the level of skill is acquired. Teams of agents can be employed to make up the learning system. A recommender system can also comprise a sub team of agents which cooperate to deliver timely information to the student. 


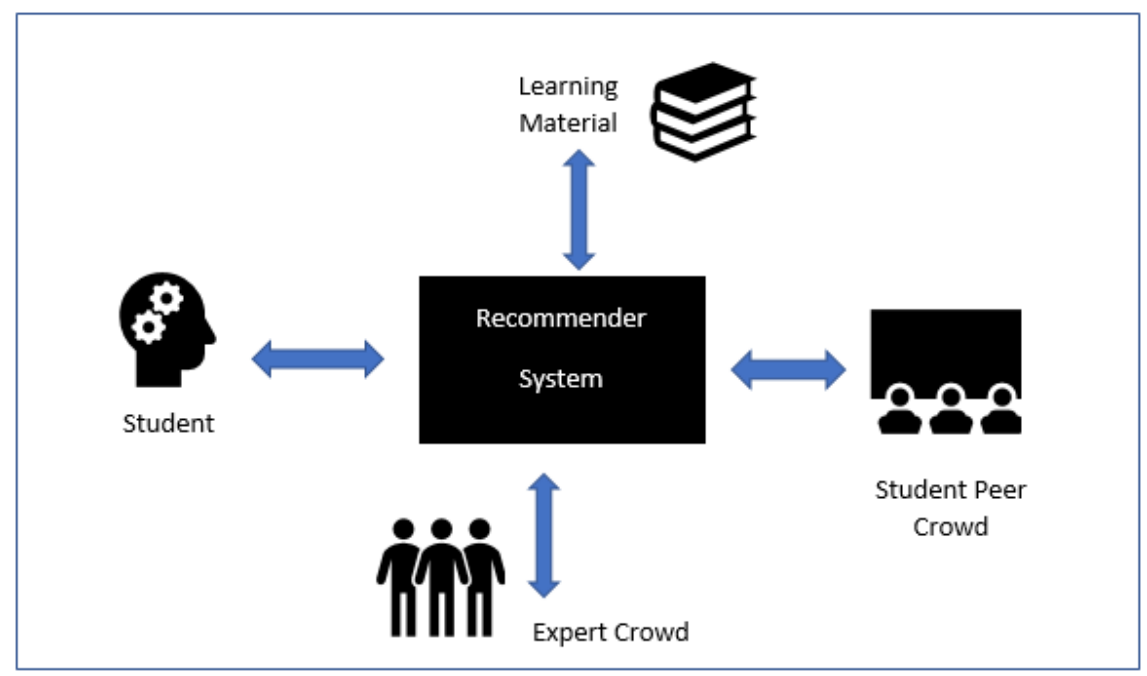

Figure 1 High Level Archtecture for Proposed Crowdsourced Recommender System

\section{Conclusion}

By creating a crowdsourced recommender system that could adapt to the needs of students individually would put the learner at the centre of learning. This would help students gain experience as they progress along with their studies and in turn collaborate with others in their learning experience. An intelligent environment will certainly help with student retention rate and additionally improve skill acquisition. A recommender system is only a small, but important, part of the e-learning eco system. Information has to be media neutral and different elements have to be combined to display the same results by different means that appeal to the user. In this paper we have put forward our arguments in favour of the use of artificially intelligent techniques to overcome specific shortcomings within e-learning systems. We strongly believe that the lack of personalization brings about a unfavourable sense of isolation that hinders rather than facilitates the learning process. The use of a recommender systems based on latest technologies to deliver personalised education material is opportune and suits all our requirements and objectives. Such a methodology further assists alleviate the issue of information overload as specifically targeted educational material will be put forward to the individual learners. In our opinion the recent developments in technology has enabled recommender systems to move to their next phase whereby networked technologies unleashed resourceful affordances that before were not possible, and that potentially they can take e-learning to its next generation. 


\section{References}

Chen, P., \& Wei, D. (2004). Multi-Agent Based Educational Environment. In F. Lin, \& M. Ally, Designing Distributed Learning Environments with Distributed Software Agents (p. 311). London: Idea Publishing Group.

Garrison, R. (2011, January 2). e-Learning in the 21st Century: A Framework for Research and Practice. Retrieved June 17, 2017, from Research Gate: researchgate.net

Jannach, D., \& Zanker, M. (2011). Recommender Systems: An Introduction. London: Cambridge University Press.

Mallia-Milanes, M., \& Montebello, M. (2017). Agent Assisted Collaborative Learning. 9th International Conference on Agents and Artificial Intelligence. Porto Portugal February 2017.

Montebello, M. (2014). Artificial Intelligence to the Rescue of e-Learning. 6th International Conference on Education and New Learning Technologies. Barcellona, Spain. . EDULearn 14.

Montebello, M. (2015). Investigating Crowd Sourcing in Higher Education. Higher Education in the Globalised Age conference. Sheffield.

Montebello, M. (2018). AI Injected e-learning (1st ed., Vol. 745). Switzerland: Springer International Publishing.

Nicholson, P. (2007). Compters and Education:Elearning from Theory to Practice. In $A$ Hybrid Intelligence-Aided Approach to Affect-Sensitive e-Learning (pp. 1-11). Springer.

Rivard, R. (2013, March 8). Measuring the MOOC Dropout Rate. Retrieved April 26, 2015, from Inside Higher Ed: www.highered.com 